\title{
Supply chain design and planning accounting for the Triple Bottom Line
}

\author{
Bruna Mota $^{\mathrm{a}^{*}}$, Ana Carvalho ${ }^{\mathrm{a}}$, Maria Isabel Gomes ${ }^{\mathrm{b}}$, Ana Barbosa-Póvoa ${ }^{\mathrm{a}}$ \\ ${ }^{a}$ CEG-IST, University of Lisbon, Av. Rovisco Pais, 1049-001 Lisboa, Portugal \\ ${ }^{b}$ CMA-FCT, Nova Universtity of Lisbon, Monte de Caparica, 2829-516 Caparica, \\ Portugal \\ bruna.mota@tecnico.ulisboa.pt
}

\begin{abstract}
In this work, a multi-objective mixed integer linear programming (moMILP) model is presented for the design and planning of sustainable closed loop supply chains. It includes strategic decisions such as facility location, definition of the transportation network, technology selection and allocation, as well as tactical decisions such as supply planning, that satisfy the demand. The first objective accounts for the economical pillar of sustainability considering profit maximization. The profit is measured through net present value (NPV) at the end of the planning horizon, accounting for costs with facilities, transportation, raw material acquisition, production and remanufacturing, inventory, product recovery and human resources. The second objective is the environmental impact minimization, evaluating the environmental pillar of sustainability. It is measured using the Life Cycle Assessment (LCA) methodology $\mathrm{ReCiPe}$, applied to the main activities of the supply chain: production, transportation and facility installation. Both unimodal and intermodal transportation are considered. The third objective accounts for the social pillar of sustainability. This is measured through socio-economic indicators applied by the European Union to its Sustainable Development Strategy. An approximation of the Pareto front is obtained through the augmented $\varepsilon$-constraint method that allows to address the trade-offs inherent to these conflicting objectives. The applicability of the model is demonstrated through a representative supply chain case study showing how this tool may help companies to adjust to the new sustainability context.
\end{abstract}

Keywords: sustainable supply chain, intermodal transportation, technology selection, environmental impact, socio-economic indicators, optimization

\section{Introduction}

The growing pressure from both customers and governments towards sustainable development is redirecting strategies focused on profit to strategies that account for the triple bottom line, where profit, planet and people are considered (Bojarski, Laínez et al. 2009). However, the complexity inherent to the integration of such decisions has been delaying the growth in both research and implementation of sustainable development practices. A number of different and interdependent factors are involved and have an impact on the economic, environmental and social performances of the supply chains, resulting in highly complex models. Facility location decisions for instance directly influence the costs and environmental impact of transportation, which is the activity responsible for the major share of both costs (Bhattacharya, Kumar et al. 2014) and environmental impact (Plambeck 2012) within supply chains. However, these decisions 
also have an impact on the community in which the facilities are located. Decisions such as the transportation network also have an impact on the three pillars of sustainability. Depending on the transportation mode chosen, the costs may be higher but compensated by a lower environmental impact or additional job creation. Within this activity there is also the possibility of selecting either unimodal transportation, in which a single transportation mode is used to distribute the product to its final destination, or intermodal transportation, where the product is carried by a combination of at least two transport modes (e.g. road, rail, sea, air) (Macharis and Bontekoning 2004). Another decision variable of significant impact is technology selection since different technologies might have different operating costs, may use different quantities of raw materials or different raw materials altogether, may result in different environmental impacts, and may require a different workforce, among many other interconnected consequences. Closing the loop by recovering end-of-life products opens an even more complex path that needs to be carefully designed and planned so as to actually translate into a more sustainable supply chain. In addition is the difficulty in quantitatively measuring such environmental and social impacts, where a significant research gap is found (Mota, Gomes et al. 2014). Hence, decision support tools that adequately address such complex problems, both at a strategic and tactical level, should be developed to help companies adjust to these current and growing sustainability pressures. This work aims to be a step forward in this direction by presenting a model for the design and planning of closed loop supply chains, which introduces the discussed decision variables and incorporates the three pillars of sustainability.

\section{Problem definition and mathematical formulation}

The proposed model aims at determining the supply chain structure, transportation network, and technology allocation, along with planning decisions that maximize profit, minimize environmental impact and maximize the social benefit of a closed loop supply chain.

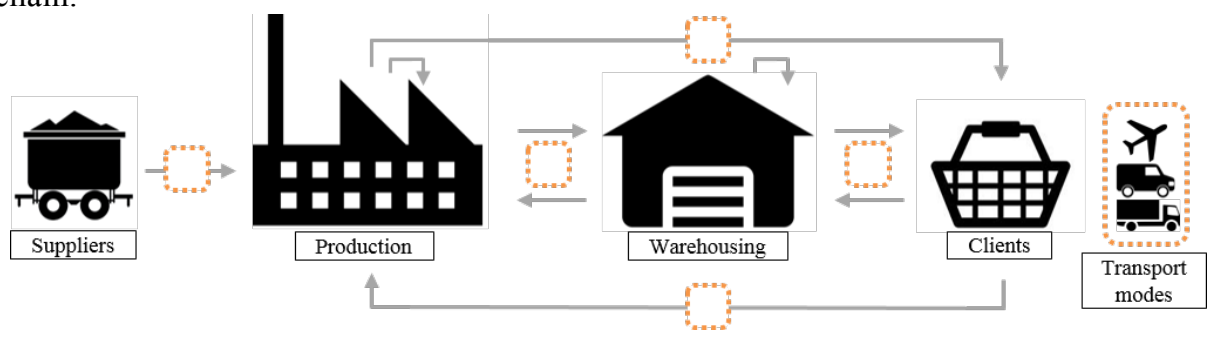

Figure 1. Modelled supply chain structure.

The modelled supply chain structure is the one depicted in Figure 1, which shows a four-echelon structure where suppliers send the raw materials to the factories, the factories transform the raw materials into final products and can then send these products to other factories, to distribution centres or directly to the clients. End-of-life products are then recovered at the clients and can be sent back to the distribution centres or directly to the factories. Transhipment is also allowed between distribution centres. In the factories the end-of-life products are remanufactured and then sold as final products. Transportation between all facilities can be performed using only one transportation mode (unimodal transportation) or using intermodal transportation. In this latter case a new entity is installed, a hub terminal, where the transfer of cargo from one transportation mode to the other is performed. The problem is modelled through a moMILP formulation. The economic objective function is obtained from the 
maximization of the NPV, as shown in equation (1), which is given by the summation of the discounted cash flows $(\mathrm{CFt})$ of each time period $\mathrm{t}$, considering the interest rate $i r$. The cash flow per time period is obtained through the difference between the net earnings (NEt) and the fraction of the total depreciable capital (FTDCt) (Eq. (1a)). At the end of the time horizon part of the total fixed capital investment $(F C I)$ - the salvage value $(s v)$ - can be recovered (Eq. (1b)).

$$
\begin{aligned}
& \max \quad N P V=\sum_{t} \frac{C F_{t}}{(1-i r)^{t}} \\
& C F_{t}=N E_{t}-F T D C_{t}, t=1, \ldots, N T-1 \\
& C F_{t}=N E_{t}-F T D C_{t}+s v \times F C I, t=N T \\
& N E_{t}=(1-t r)\left[\sum_{m, i, j:(m, i, j) \in F_{I N C F P}} p s u_{m} \sum_{a} X_{m a i j t}\right. \\
& -\left(\sum_{m, i, j:(m, i, j) \in F_{\text {OUTSUPRM }}} r m c_{m} \sum_{a \in A} X_{m a i j t}\right. \\
& +\sum_{m, g, i:(m, g) \in G_{P R O D} \wedge i \in I_{f}} o p c_{g} \Psi_{m g i t} \\
& +\sum_{m, i, j:(m, i, j) \in F_{\text {OUTCRP }}} r p c_{m} \sum_{a \in A} X_{\text {maijt }} \\
& +\sum_{m, g, i:(m, g) \in G_{R E M} \wedge i \in I_{f}} o p c_{g} \Phi_{m g i t} \\
& +\sum_{a, m, i, j:(a, m, i, j) \in N e t P \wedge a \in A_{\text {plane }}} t c_{a} \cdot p w_{m} \cdot d_{i j} \cdot X_{\text {maijt }} \\
& +\sum_{a, m, i, j:(a, m, i, j) \in N e t P \wedge a \in A_{\text {truck }}} v t c_{a} \cdot d_{i j} \cdot Q_{a i j t}
\end{aligned}
$$

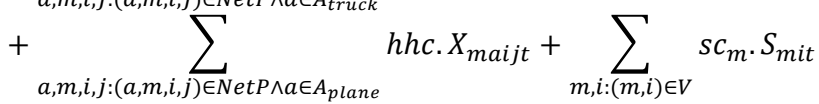

$$
\begin{aligned}
& +\sum_{i \in I_{f} \cup I_{w} \cup I_{\text {air }}} w_{i} \cdot l c_{i} \cdot m w h . Y_{i} \\
& +\sum_{m, g, i:(m, g) \in G_{P R O D} \cup G_{R E M}} w_{g} \cdot l c_{i} \cdot m w h \cdot \mathrm{z}_{g m i} \\
& \left.\left.+\sum_{a, i, j:(a, i, j) \in N e t} w_{a i j} \cdot l c_{i} \cdot m w h . K_{a i j}\right)\right]+\operatorname{tr} \cdot D P_{t}
\end{aligned}
$$

$$
D P_{t}=\frac{(1-s v) \cdot F C I}{N T}
$$




$$
\begin{aligned}
& F T D C_{t}=\frac{F C I}{N T} \\
& F C I=\sum_{i \in I_{f} \cup I_{w}} e c_{i} \cdot Y_{i}+\sum_{m, g, i:(m, g) \in G_{P R O D} \cup G_{R E M} \wedge I_{f}} t e c_{g} \cdot \mathrm{Z}_{g m i} \\
& +\sum_{a, i, j: a \in A_{\text {truck }} \wedge(a, i, j) \in N e t} f t c_{a} \cdot \frac{\mathrm{K}_{a i j}}{2}+\sum_{i \in I_{\text {air }}} h u b_{i} Y_{i}
\end{aligned}
$$

The net earnings (NEt) are given by the difference between the incomes, defined by the amount of products sold, and the total cost per time period (Eq. (1c)). The costs include the costs with raw materials (first term, where $X_{\text {maijt }}$ is the flow of product $m$ through transportation mode $a$ between entities $i$ and $j$ in time period $t$ ), production (second term, where $\Psi_{m g i t}$ is the amount of product $m$ produced through technology $g$ in entity $i$ in time period $t$ ), product recovery (third term), remanufacturing (fourth term, where $\Phi_{\text {mgit }}$ is the amount of product $m$ remanufactured through technology $g$ in entity $i$ in time period $t$ ), transportation costs for air (fifth term) and for road transportation (sixth term, where $Q_{a i j t}$ is the number of trips with transportation mode $a$ between entities $i$ and $j$ in time period $t$ ), handling costs at the hub terminal (seventh term), inventory costs (eighth term, where $S_{m i t}$ is the inventory of product $m$ in entity $i$ in time period $t$ ) and labour costs at entities (ninth term, where $Y_{i}$ is a binary variable for entity installation in location $i$ ), at production and remanufacturing technologies (tenth term, where $\mathrm{Z}_{g m i}$ is a binary variable for technology allocation) and at transportation (eleventh term, where $K_{a i j}$ is a binary variable for transportation link establishment). The last term describes the depreciation of the capital invested $(D P t)$ for which the straight-line method is assumed, as described by Cardoso et al. (2013) and as shown in Eq. (1d). Through Eq. (1e) it is assumed that the payment of the fixed capital investment is divided into equal sums for each time period. The fixed capital investment $(F C I)$ is defined in Eq. (1f) and is given by the investment in facilities (first and last term), investment in production and remanufacturing technologies (second term) and investment in transportation links (third term).

$$
\begin{aligned}
\min \quad \text { EnvImpact } & =\sum_{c}\left(\sum_{t \in T} \sum_{m, g, i:(m, g) \in G_{P R O D} \cup G_{R E M} \wedge I_{f}} I_{m g c}\left(\Psi_{m g i t}+\Phi_{m g i t}\right)\right. \\
& \left.+\sum_{t \in T} \sum_{a, m, i, j:(a, m, i, j) \in N e t P} I_{a c} p w_{m} d_{i j} X_{m a i j t}+\sum_{i \in I_{f \cup I_{w}}} I_{i c} a_{i} Y_{i}\right) \eta_{c}
\end{aligned}
$$

The environmental objective function is modelled using the ReCiPe methodology as described in Mota et al. (2014). For each midpoint category $c$ the environmental impact of production (first term), transportation (second term) and entity installation (third term) is determined, summed and normalized as shown in Eq. (2).

The social objective function is designed taking into account socio-economic indicators applied by the European Union to its Sustainable Development Strategy, namely, the Gross Domestic Product (GDP) and the unemployment rate of each country. These two indicators are applied through $\mu_{i}$, a regional factor. Hence, two social objective functions are in fact given. One where $\mu_{i}$ results from GDP statistics and the other where the regional factor results from unemployment rate statistics. This way the 
models prefers the location of entities (first term), the allocation of technologies (second term) and the establishment of transportation links (third term) in regions of higher unemployment rate or of lower GDP, according to the selected regional factor. Additionally these social objective functions are designed to balance these regional indicators with the maximization of the number of jobs created $\left(w_{i} / w_{g} / w_{a i j}\right)$, as shown in Eq. (3).

min SocialAssessment

$$
\begin{aligned}
& =\sum_{i \in I_{f} \cup I_{w} \cup I_{a i r}} \mu_{i} \frac{Y_{i}}{w_{i}}+\sum_{m, g, i:(m, g) \in G_{P R O D} \cup G_{R E M} \wedge i \in I_{f}} \mu_{i} \frac{Z_{g m i}}{w_{g}} \\
& +\sum_{a, i, j:(a, i, j) \in N e t} \mu_{i} \frac{K_{a i j}}{w_{a i j}}
\end{aligned}
$$

Given the conflicting objectives a multiobjective methodology is implemented to obtain an approximation of the Pareto front. The augmented $\mathcal{E}$-constraint method is applied as described in Mavrotas et al. (2009).

\section{Case-study and results}

The developed model was applied to a European case-study where decisions regarding facility location, technology selection and allocation as well as transportation network establishment are to be taken so as to satisfy the demand. Two possible locations exist for factories, one in the United Kingdom and one in Italy, where the suppliers are already located. Seven possible locations exist for warehouses: United Kingdom, Italy, Spain, Germany, Portugal, Hungary and Bulgaria. Three airports for intermodal transportation: one in France, one in Spain and one in Belgium. The targeted markets are located in Portugal, Spain, Italy, the United Kingdom and Germany. Two final products are modelled. Each can be obtained through production or remanufacturing. For product 1 there are two alternative production technologies and one for remanufacturing. For product 2 there is one production and one remanufacturing technology. All technologies are characterized by different costs, capacities, environmental impacts and number of workers. There are three transport modes available, two types of trucks and one by airplane, again with different attributes. In case a hub terminal is installed, it is also characterized in terms of economic, environmental and social performance. Supply capacity, flows of products at entities, inventory levels, production and remanufacturing capacities, and transportation capacities are also constrained.

When maximizing NPV the model installs two factories, one in the United Kingdom and one in Italy (which are selected in all scenarios), and two warehouses, one in Bulgaria and one in Hungary. For production of product 1 a technology is chosen which even though having higher installation costs, has a higher production capacity and lower operating costs. Both types of trucks are used in a solution that balances their capacities with the transportation costs. The highest share of costs results from human resources, followed by transportation. In fact the model chooses to minimize the total number of workers and to place them in the countries with the lower hourly labour costs. When minimizing environmental impact the model chooses to install two warehouses, in the United Kingdom and Portugal. Product recovery is increased and remanufacturing is maximized to full capacity. The same technology as the one selected in the previous 
scenario is used since it also has the lowest environmental impact. To reduce the environmental impact of transportation, which is the highest after that of production, the model chooses to perform intermodal transportation using all three airports. This solution translates in a $47 \%$ NPV reduction and a $40 \%$ increase in the number of workers. When optimizing for maximum social benefit using the regional factor related to GDP the model returns once again the warehouses in Bulgaria and Sofia, the countries with the lowest GDP. At the same time it selects the technology and transportation options that maximize the number of jobs created in a compromise that minimizes the social indicator. When using the regional factor related to unemployment rate the same takes place but the warehouses are located in Spain and in Portugal, the countries with the highest unemployment rate. This translates in a significant economic performance deterioration.

\section{Conclusions}

The presented work proposes a decision support tool to study how sustainability can be introduced in supply chain design and planning. NPV is used to measure the economic performance of the supply chain. The LCA methodology ReCiPe is used as an environmental impact indicator. GDP and unemployment rate are introduced in social indicators that balance job creation in regions with lower socio-economic performances with the maximization of job creation. The model allows to understand the impact of decisions such as facility location, technology selection and allocation, and transportation network establishment, on the three pillars of sustainability. Through its application to a European case-study results show how the different indicators return conflicting solutions, as expected. Multiobjective optimization helps to visualize the compromise between the different indicators (results not shown). Considering that economic incentives could be provided to maintain economic performance, the results still raise the question of what is more important, the environmental or the social performance? And within social performance, is it preferable to maximize job creation or to have job creation in regions with lower socio-economic conditions? And which social indicator is more suitable? Future work should further extend the model to include additional decision variables that have a direct impact on the performance of the supply chain and affect its sustainability. Further case-studies will also contribute to a better understanding of the implications of the proposed environmental and social indicators.

\section{References}

Bhattacharya, A., S. A. Kumar, et al. (2014). "An intermodal freight transport system for optimal supply chain logistics." Transportation Research Part C: Emerging Technologies 38(0): 73-84.

Bojarski, A. D., J. M. Laínez, et al. (2009). "Incorporating environmental impacts and regulations in a holistic supply chains modeling: An LCA approach." Computers \& Chemical Engineering 33(10): 1747-1759.

Cardoso, S. R., A. P. F. Barbosa-Póvoa, et al. (2013). "Design and planning of supply chains with integration of reverse logistics activities under demand uncertainty." European Journal of Operational Research 226(3): 436-451.

Macharis, C. and Y. M. Bontekoning (2004). "Opportunities for OR in intermodal freight transport research: A review." European Journal of Operational Research 153(2): 400-416.

Mavrotas, G. (2009). "Effective implementation of the $\varepsilon$-constraint method in multi-objective mathematical programming problems." Applied Mathematics and Computation 213(2): 455-465.

Mota, B., M. I. Gomes, et al. (2014). "Towards supply chain sustainability: economic, environmental and social design and planning." Journal of Cleaner Production. 
Plambeck, E. L. (2012). "Reducing greenhouse gas emissions through operations and supply chain management." Energy Economics 34, Supplement 1(0): S64-S74. 